This item was submitted to Loughborough's Institutional Repository (https://dspace.lboro.ac.uk/) by the author and is made available under the following Creative Commons Licence conditions.

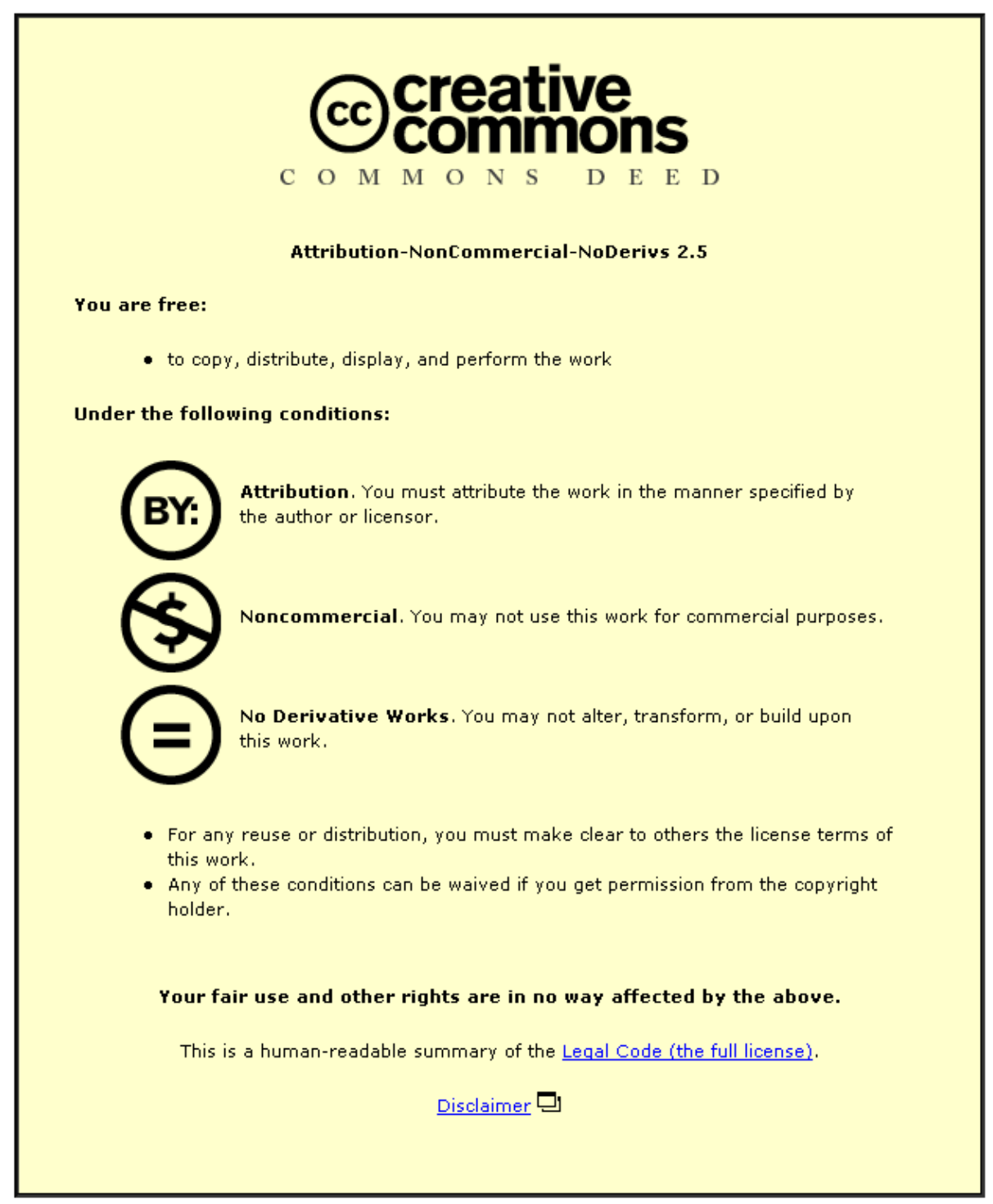

For the full text of this licence, please go to: http://creativecommons.org/licenses/by-nc-nd/2.5/ 


\section{Cutaneous Sensibility and Peripheral Nerve Function in Patients with Unmedicated Essential Hypertension}

Louisa Edwards ${ }^{\mathrm{a}}$, Christopher Ring ${ }^{\mathrm{a}}$, David McIntyre ${ }^{\mathrm{a}}$, John. B Winer ${ }^{\mathrm{b}}$ \& Una Martin ${ }^{\mathrm{c}}$

${ }^{a}$ International Centre for Health and Exercise Research, University of Birmingham, Birmingham. B15 2TT. U.K.

${ }^{\mathrm{b}}$ Clinical Neurophysiology, University Hospital, Birmingham. B15 2TH. U.K.

${ }^{\text {c }}$ School of Medicine, University of Birmingham, Birmingham. B15 2TH. U.K.

Running head: Peripheral Nerve Function in Essential Hypertension

Corresponding Author: Dr Louisa Edwards, International Centre for Health and Exercise Research, University of Birmingham, Birmingham. B15 2TT. UK. Tel: +44 1214158785. Fax: +44 121414 4121. E-mail: L.Edwards@bham.ac.uk. 


\begin{abstract}
Sensorimotor deficits in patients with essential hypertension may be due to impaired nerve function. Cutaneous sensory thresholds, median nerve sensory and motor conduction velocities and median nerve sensory action potential amplitudes were assessed in 30 patients with unmedicated essential hypertension and 29 normotensives. Cutaneous sensory thresholds were higher and sensory action potential amplitudes smaller in hypertensives than normotensives whereas sensory and motor nerve conduction velocities did not differ between groups. These data suggest that hypertension may reduce the number of active sensory nerve fibres without affecting myelination. Sensory action potential amplitudes were inversely related to cutaneous sensory thresholds suggesting that subclinical axonal neuropathy of sensory afferents may help account for perceptual deficits that characterise hypertension.
\end{abstract}

Descriptors: Cutaneous sensory thresholds, Essential hypertension, Median nerve, Nerve conduction velocity, Sensory action potential amplitudes. 


\section{Introduction}

Hypertension is associated with the development of neuropathy (Tesfaye et al., 2005) and microvascular disease (Forrest, Maser, Pambianco, Becker, \& Orchard, 1997) in diabetes mellitus. Diabetic polyneuropathy is associated with progressive axonal degeneration (Dyck et al., 1986), and demyelination (Thomas \& Lascelle, 1965). In polyneuropathy, microvascular abnormalities in epineural and endoneuronal vessel walls, including basement membrane thickening, endothelial cell proliferation and vessel occlusions (Ibrahim et al., 1999; Tesfaye et al., 1993), impede oxygen transport and change the blood/nerve barrier leading to ischaemic and hypoxic damage of peripheral nerves (Teunissen et al., 2002).. Although the aetiology of polyneuropathy is multifactorial, hypertension has been identified as a risk factor (Forrest et al., 1997; Zarrelli et al., 2001). The frequent coexistence of hypertension and polyneuropathy in diabetes mellitus suggests a vascular basis for the onset and progression of diabetic neuropathy due to progressive microvascular insufficiency (Malik, 2000). Treatment with angiotensin converting enzyme inhibitors, that reduce blood pressure by vasodilatation, improved nerve conduction velocities in diabetics (Malik et al., 1998; Reja, Tesfaye, Harris, \& Ward, 1995). Further, pulse pressure has been reported to be a strong independent predictor of nerve conduction velocity in patients with diabetes mellitus (Jarmuzewska \& Mangoni, 2005).

Despite evidence for an association between hypertension and neuropathy in diabetics, the influence of hypertension on nerve function in the absence of diabetes has yet to be established. Hypertension is a risk factor for peripheral vascular disease (Makin, Lip, Silverman, \& Beevers, 2001). Peripheral vascular disease is associated with structural alterations in the microcirculation, with remodelling of the microvasculature leading to reductions in the number of arterioles or capillaries in the vascular beds of target organs 
(Mourad \& Laville, 2006). The vascular system supplying the peripheral nervous system, which incorporates the vasa nervorum, lacks autoregulatory capabilities (Smith, Kobrine, \& Rizzoli, 1977). This absence of autoregulation makes the peripheral nerves vulnerable to hypoxia when their blood supply is compromised (Low \& Tuck, 1984; Olsson, 1972). Thus, it is plausible that hypertensives, characterised by increased vascular resistance, are more vulnerable to ischaemia and hypoxia of the peripheral nerves.

Evidence from animal studies demonstrates that the vascular supply to the peripheral nerves, caused by thickening of the arterial wall and luminal narrowing of interfascicular arteries, is impaired in the spontaneously hypertensive rat (Sabbatini, Bellagamba, Vega, \& Amenta, 2001; Sabbatini, Vega, \& Amenta, 1996). Further, antihypertensive treatment partially reversed these changes (Sabbatini et al., 2001). In the spontaneously hypertensive rat, morphological changes have been documented in the aortic depressor nerve (Fazan, Fazan, Salgado, \& Barreira, 1999) (Fazan, Salgado, \& Barreira, 2001) and sciatic nerve (Tomassoni, Traini, Vitaioli, \& Amenta, 2004) that were accompanied by decreased nerve conduction velocity. Importantly, these morpological changes were not found when blood pressure was maintained at normotensive levels by treatment with the vasodilator, hydralazine (Tomassoni et al., 2004). Overall, these animal studies provide further evidence that hypertension is a risk factor for peripheral neuropathy.

Evidence from nerve function studies in human hypertension is less consistent. A reduction in motor nerve conduction velocities in the upper extremities has been reported in hypertensives compared to normotensives (Viskoper, Chaco, \& Aviram, 1971). This study also noted an inverse relationship between nerve conduction velocity and diastolic blood pressure (Viskoper et al., 1971). However, subsequent studies failed to replicate this original 
report, instead they found normal sensory and motor nerve conduction velocities in the upper and lower limbs of hypertensive patients (Bridgman, Bidgood, \& Hoole, 1973; Halar, Stewart, Venkatesh, \& Chrissian, 1978). These inconsistent reports may be explained by methodological differences among studies. Nerve conduction measurements vary with limb temperature, which Viskoper et al (1971) did not measure, in contrast to the latter null studies (Bridgman et al., 1973; Halar et al., 1978). In addition, none of these studies controlled for antihypertensive medication (Bridgman et al., 1973; Halar et al., 1978; Viskoper et al., 1971), which may have had a beneficial effect on nerve function (Malik et al., 1998). Accordingly, the present study investigated the influence of essential hypertension on peripheral nerve function in unmedicated, newly-diagnosed patients with essential hypertension uncomplicated by diabetes mellitus or symptoms of peripheral neuropathy. It was hypothesised that hypertensives would show deficits in nerve function, reminiscent of those reported in diabetic polyneuropathy. As previous studies only investigated nerve conduction velocity, the current study measured sensory action potential amplitudes in addition to nerve conduction velocities to investigate the possibility that hypertension may cause axonal degeneration as well as demyelination.

Evidence has established that hypertension is characterized by reduced sensibility to peripheral stimulation (Ghione, 1996; Waldstein, Manuck, Ryan, \& Muldoon, 1991). Studies have demonstrated that hypertensives have reduced perception of pain (Ghione, 1996). Higher sensory detection thresholds have also been reported in hypertensives compared to normotensives using electrical stimulation of the tooth pulp (Ghione et al., 1985; Ghione, Rosa, Mezzasalma, \& Panattoni, 1988; Rosa, Ghione, Panattoni, Mezzasalma, \& Giuliano, 1986; Zamir \& Shuber, 1980) as well as electrocutaneous stimulation of the hand (Rosa, Vignocchi, Panattoni, Rossi, \& Ghione, 1994). In addition, compromised visual perception 
has also been reported in hypertensives (Mazzucchi et al., 1986; Shapiro, Miller, King, Ginchereau, \& Fitzgibbon, 1982). In sum, there is preliminary evidence for perceptual deficits in hypertension. The present study investigated the influence of essential hypertension on electrocutaneous sensory thresholds. It was hypothesised that hypertensives would show higher sensory thresholds, consistent with the one previous study which assessed electrocutaneous sensibility in hypertension (Rosa et al., 1994). Thus, one of the aims of the present study was to replicate Rosa et al.'s (1994) preliminary observations. To our knowledge, no previous study has examined both peripheral nerve function and cutaneous sensory thresholds in essential hypertensives. The current study assessed the relationship between sensory nerve function and cutaneous sensibility in hypertensives to investigate the possibility that sensory deficits in hypertension may be explained by changes in sensory nerve function.

\section{Methods}

\section{Participants}

Thirty patients with essential hypertension and 29 healthy normotensive individuals participated. Table 1 shows the characteristics of the hypertensive and normotensive groups. Patients with newly diagnosed essential hypertension were recruited from the hypertension clinic at University Hospital, Birmingham, UK, and were tested prior to the initiation of pharmacological treatment. Normotensive volunteers were recruited from the general population of Birmingham, UK, and screened in the same way as the hypertensive group. Participants were instructed to refrain from caffeine, alcohol, and vigorous exercise for 2 hours prior to testing. The study was conducted in accordance with the Declaration of Helsinki and approved by the local ethics committee. Volunteers gave written consent to participate. 


\section{Screening}

Exclusion Criteria. In an initial screening session, each participant's medical status and eligibility were determined. Participants were excluded for the following: age younger than 18 years or older than 50 years, current use of prescription medication (excluding contraceptives), any chronic disease or any condition predisposing to carpal tunnel syndrome or peripheral neuropathy including diabetes mellitus (either pre-existing or diagnosed on random blood sugar sample), excess alcohol intake ( $>28$ units (1 unit = $284 \mathrm{ml}$ of beer, 125 $\mathrm{ml}$ of wine, or $25 \mathrm{ml}$ of spirits) of alcohol per week in men, $>21$ units of alcohol per week in women), thyroid disease, chronic liver disease, cerebrovascular disease, peripheral vascular disease, rheumatoid or osteoarthritis, obesity, acromegaly or gout, symptoms of numbness, tingling, itching or abnormal sensations, neuromuscular disease, peripheral nerve injury, hereditary/genetic neuropathy, neck or back surgery, cancer or chemotherapy, history of myocardial infarction, symptoms of angina or major psychiatric disorder (e.g., depression, schizophrenia). If clinically indicated the patients in the hypertensive group had appropriate investigations to exclude secondary causes of hypertension before taking part in the study. On the basis of these investigations patients were excluded if there was evidence of underlying renal or adrenal pathology including renal artery stenosis, glomerulonephritis, pyelonephritis, Conn’s syndrome or phaeochromocytoma.

Blood Pressure Status. British Hypertension Society guidelines were used to establish blood pressure status (Williams et al., 2004). Each participant's blood pressure was measured at the non-dominant upper arm for 24 hours using an ambulatory blood pressure monitor (Model 90217, SpaceLabs Medical). Participants were instructed to go about their usual activities during the monitoring period. Blood pressure readings were obtained every 30 minutes 
between the hours of 7:00 a.m. and 11:00 p.m., and every 60 minutes between 11:00 p.m. and 7:00 a.m. Mean daytime systolic and diastolic blood pressures were calculated from all readings taken between 7:00 a.m. and 11:00 p.m. Patients with a systolic blood pressure of $\geq 160 \mathrm{mmHg}$ or a diastolic blood pressure of $\geq 100 \mathrm{mmHg}$ at referral, and confirmed at clinic and on ambulatory blood pressure monitoring (mean daytime pressure) were diagnosed as hypertensive; this category comprised $71 \%$ of patients. Patients with a systolic blood pressure of 140-159 mmHg and/or a diastolic blood pressure of 90-99 mmHg at referral, clinic, and on ambulatory blood pressure monitor, were diagnosed as hypertensive if their 10 year coronary heart disease risk was $\geq 15 \%$ and/or there was evidence of left ventricular hypertrophy on a 12 lead electrocardiograph or echocardiograph, or there was other evidence of end organ damage. The cardiovascular risk profile was calculated using the Joint British Societies Cardiac Risk Assessor computer program (Wood et al., 1998). Participants were classified as being normotensive if they had a clinic systolic blood pressure of $<140 \mathrm{mmHg}$ and a clinic diastolic blood pressure of $<90 \mathrm{mmHg}$, confirmed on ambulatory blood pressure monitoring, and $<15 \%$ risk of coronary heart disease in the next 10 years. In addition, laboratory blood pressure and heart rate were measured using an oscillometric sphygmomanometer (Dinamap, Critikon) and a brachial cuff attached to the participant's upper left arm.

\section{Apparatus and Measurements}

For the Cutaneous sensory threshold procedure, a control box with red and green light emitting diodes and buttons marked "no" and "yes" was used to register the participant’s responses. A computer was programmed in Spike2 (CED) to record responses and present stimuli using a Power1401 (CED). Cutaneous stimuli (1 ms square wave pulses at $250 \mathrm{~Hz}$ for $60 \mathrm{~ms}$ ) were delivered electrocutaneously using a constant current stimulator (DS7A, 
Digitimer) via a surface electrode secured to the index finger of the dominant hand. The 250 $\mathrm{Hz}$ frequency was chosen as it has been previously shown to stimulate both $\mathrm{A} \beta$ and $\mathrm{A} \delta$ nerve fibres (Katims, Naviasky, Rendell, Ng, \& Bleecker, 1987). A maximum stimulation intensity of $4 \mathrm{~mA}$ was employed to prevent stimulation of nociceptive A $\delta$ fibres (Ring et al., in submission). The stimulating electrode comprised two $10 \mathrm{~mm}$ stainless steel disks (Nicolet) secured with tape on the skin of the dorsolateral surface of distal phalanges with the anode medial. The finger site was cleaned to reduce impedance below $100 \mathrm{k} \Omega$.

Sensory action potentials $(\mu \mathrm{V})$ were recorded antidromically from the median nerve at the palmar side of the index finger of the dominant hand. The most proximal electrode contact was placed at the proximal finger joint using a single differential surface electrode (DE 2.1, Delsys), with electrode contacts adapted to have a $3 \mathrm{~cm}$ interelectrode distance. A separate ground electrode was fixed to the ipsilateral wrist bone. The sensory action potential signal was bandpass filtered (20-450 Hz) and amplified (gain = 10, 000) using a Bagnoli-2 amplifier (Delsys), and recorded at 10,000 Hz. Electromyographic activity associated with the compound motor nerve action potentials $(\mathrm{mV})$ was recorded orthodromically from the abductor pollicis brevis midway between the thumb joint and the wrist crease using a single differential surface electrode with $1 \mathrm{~cm}$ central spacing (DE 2.1, Delsys), referenced to an electrode located on the ipsilateral wrist bone. The electromyographic signal was bandpass filtered (20-450 Hz), amplified (gain = 100) using a Bagnoli-2 amplifier (Delsys), and recorded at $5000 \mathrm{~Hz}$.

The median nerve was stimulated by an electrocutaneous stimulus (single $100 \mu \mathrm{s}$ square wave pulse) delivered to the skin over the median nerve at both the proximal wrist crease, between the central palmaris longus tendon the flexor capri radialis tendon as well as 
at the elbow crease at the medial aspect of the cubital fossa and biceps tendon. Stimuli were delivered using the constant current stimulator and bar electrode. This bar electrode comprised two $5 \mathrm{~mm}$ saline soaked felt contacts with $3 \mathrm{~cm}$ spacing (Nicolet) secured with tape on the skin of the wrist and elbow with the cathode distal. Impedance at the wrist and elbow sites was kept below $8 \mathrm{k} \Omega$.

Participants sat upright with their dominant arm resting on a table. During the sensory threshold assessment the dominant hand rested, palm down, on the response box. During the nerve function testing the dominant hand was held in a relaxed position, palm upwards, with the forearm supinated. Skin surface temperature of the dominant hand and arm was maintained between $31-35{ }^{\circ} \mathrm{C}$, using a large gel heat pack (Harvard Apparatus). Skin surface temperature was measured before each assessment using an infrared thermometer (3000 A First Temp Genius, Sherwood Davis \& Geck).

\section{Procedure}

Height and weight were measured, and then participants sat and relaxed during a formal rest period (5 min) while their blood pressure and heart rate were measured during minutes 1,3 , and 5. Participants then completed questionnaires measuring health behaviours and demographics. Following instrumentation and instruction (20 min), their cutaneous sensory threshold was determined (15 minutes) using an adaptive up-down staircase procedure (Levitt, 1971). Participants then completed auditory, tactile, and visual simple reaction time tasks (60 min) that were separated by 5 min rest periods (data reported in Edwards, Ring, McIntyre, Carroll, \& Martin, 2007). Finally, median nerve function was assessed (30 min).

\section{Cutaneous Sensory Thresholds}


Three cutaneous sensory thresholds were determined concurrently by interleaving three updown staircases (Levitt, 1971). Stimulation comprised a series of five square-wave pulses of $1 \mathrm{~ms}$ duration at $250 \mathrm{~Hz}$. A green warning light was illuminated to signify the beginning of each trial, while a red light signified the end of the trial. Participants were told that the stimulus could occur at any point between the green light illumination and the beginning of the red light illuminating. At the end of each trial the participant pressed either a "YES" button, if they perceived a stimulation, or a "NO" button, if they did not detect a sensation. Participants were told that stimulation would elicit a tingling sensation, and moreover, they were also told that the sensation would never feel painful. Indeed, participants were asked to report if stimulation became painful at any time. Crucially, none of the participants reported feeling any pain associated with the stimulation. At a frequency of $250 \mathrm{~Hz}$, and a mean intensity of $0.59 \mathrm{~mA}$, the stimulus characteristics were expected to stimulate both $\mathrm{A} \beta$ and $\mathrm{A} \delta$ fibres. Importantly, at the very low levels of electocutaneous stimulation used here, coupled with pain never being reported, it is unlikely that nociceptive A $\delta$ fibres were activated. On the first trial of each staircase, the stimulus intensity was $0 \mathrm{~mA}$. For each staircase, stimulus intensity was increased in $1 \mathrm{~mA}$ steps until the participant first detected a sensation (first reversal), and then decreased in $0.4 \mathrm{~mA}$ steps until the participant no longer detected a sensation (second reversal). Each staircase then continued in $0.1 \mathrm{~mA}$ steps until the three staircases had completed three further ascending and descending series (i.e., six more reversals). The $50 \%$ cutaneous sensory threshold (mA) was defined as the average of the peaks and troughs during the second and third series (i.e., third, fourth, fifth and sixth reversal points) of each staircase. The inter-trial interval was $3 \mathrm{~s}$.

\section{Median Nerve Function}


The electrodes for recording the sensory action potential and compound motor action potentials were attached. Next, the optimal location for stimulating the median nerve at the wrist was found by slowly moving the stimulating electrode around the approximate site while a computer program delivered a series of $10 \mathrm{~mA}$ electrocutaneous stimulations every 4 s. The site causing the greatest motor twitch was identified and selected. The participant was then asked to relax while a $10 \mathrm{~mA}$ stimulation was delivered. The resultant sensory and compound motor action potentials were immediately displayed on the computer screen. Stimulation intensity was then increased in $4 \mathrm{~mA}$ steps. Stimulation intensity increased in 4 mA steps until the amplitude and latencies of both the sensory and compound motor action potentials became supramaximal. Participants' limb temperature was checked throughout, and if the temperature fell below $31^{\circ} \mathrm{C}$, the hand was warmed and the procedure repeated. This determination of the intensity required to produce a supramaximal median nerve stimulation was then repeated at the elbow.

The participant then relaxed while a computer program delivered electrocutaneous stimuli at the predetermined supramaximal intensity for 12 trials. The sensory action potential and compound motor action potentials were recorded concurrently. Waveforms were averaged together to create a grand average waveform for both sensory and compound motor action potentials. This procedure was repeated three times. The interstimulus interval was 7-s. The stimulating electrode was then removed from the wrist and the site of the cathode was marked. The temperature of the upper limb was checked immediately prior to and following this procedure to help maintain skin temperature between $31-35^{\circ} \mathrm{C}$. The procedure was then repeated three times at the elbow. After the procedure was repeated at the elbow site, three measurements were obtained: a) distance between the elbow and wrist stimulating sites, b) distance between each stimulating electrode site and the proximal electrode contact at the 
sensory action potential recording site, c) circumference for index finger at the site of the sensory action potential recording electrode proximal electrode contact.

\section{Data Reduction and Analysis}

The reliability of measurement was determined by calculating intraclass correlation coefficients among the three measures of cutaneous sensory thresholds, nerve conduction velocities and sensory action potentials. These analyses revealed high test-retest reliability for the sensory thresholds (ICC $=.993, p<.001$ ), and, therefore, the three cutaneous sensory thresholds were averaged to create a grand mean cutaneous sensory threshold for each participant. Action potential latencies (ms) were measured from the onset of the negative deflection. Sensory action potential amplitudes were measured from the onset to the peak of the negative deflection $(\mu \mathrm{V})$. Nerve conduction velocities $(\mathrm{m} / \mathrm{s})$, for both the sensory action potential and compound motor action potentials, were calculated from the difference in latency between wrist and elbow stimulations (ms) divided by the distance from elbow to wrist $(\mathrm{m})$. Test-retest reliabilities were high for motor $(\mathrm{ICC}=.979, p<.001)$ and sensory $($ ICC $=.950, p<.001)$ nerve conduction velocity assessments. Similarly, the sensory action potential amplitude measurements were reliable for both wrist (ICC $=.999, p<.001)$ and elbow (ICC $=.997, p<.001)$ sites. Accordingly, the three assessments for each of these nerve function measures were averaged. The set of three resting laboratory blood pressure recordings were averaged to provide measures of mean resting laboratory systolic blood pressure, diastolic blood pressure and heart rate.

Separate 2 Group (hypertensive, normotensive) analyses of covariance (ANCOVAs) were performed on cutaneous sensory thresholds, sensory and compound motor nerve conduction velocities and sensory action potential amplitudes. Age (years), body mass index 
$\left(\mathrm{kg} / \mathrm{m}^{2}\right)$ and sex were entered as covariates because these factors can affect nerve function measurements (Bolton \& Carter, 1980; Buschbacher, 1998; Schaumburg, Spencer, \& Ochoa, 1983). In addition, index finger circumference ( $\mathrm{mm}$ ) and stimulation-to-recording distance (mm) were also entered as covariates for sensory action potential amplitudes analyses because of their known effects on sensory action potentials (Bolton \& Carter, 1980; Horowitz \& Krarup, 1992). Eta-squared $\left(\eta^{2}\right)$, a measure of effect size, is reported. Regression analyses were used to determine the association between sensory action potential amplitudes and cutaneous sensory thresholds, after controlling for the potentially confounding effects of age, sex, body mass index, finger circumference and stimulation-to-recording distance. A significance level of .05 was adopted. Differences in the reported degrees of freedom reflect occasional missing data. The data were analysed using SPSS 14.0.

\section{Results}

\section{Group Characteristics}

Group blood pressures and demographics are presented in Table 1. Chi-square analysis revealed no significant differences for sex, $\chi^{2}(1)=1.36, p=.24$, between the hypertensive group (18 men, 12 women) and the normotensive group (13 men, 16 women). Similarly, smoking status, $\chi^{2}(1)=2.59, p=.11$, did not differ between the hypertensive group (22 nonsmokers, 8 smokers) and the normotensive group (26 non-smokers, 3 smokers). A series of 2 Group (hypertensive, normotensive) analyses of variance (ANOVAs) were performed on the continuous variables (see Table 1). These analyses confirmed that, compared to the normotensive group, the hypertensive group exhibited higher blood pressures and heart rates. Hypertensives were also older and had a higher body mass index than normotensives. The groups did not differ in terms of alcohol consumption. Because age, body mass index and sex 
are known to influence peripheral nerve function (Bolton \& Carter, 1980; Buschbacher, 1998; Schaumburg et al., 1983), and because both age and body mass index were different between blood pressure groups, these variables were adjusted for in the analyses below.

Separate 2 Group (hypertensive, normotensive) ANOVAs were performed on variables that have previously been shown to affect peripheral nerve function measures, including limb temperature $\left({ }^{\circ} \mathrm{C}\right)$, room temperature $\left({ }^{\circ} \mathrm{C}\right)$, index finger circumference $(\mathrm{mm})$, and intensity of supramaximal wrist and elbow stimulation (mA). In all instances, there was no difference between groups for any of these variables. However, because of the wellknown influence of finger circumference (Bolton \& Carter, 1980) and stimulation-torecording distance (Horowitz \& Krarup, 1992) on the amplitude of sensory action potentials, these variables were adjusted for in the analyses of sensory amplitudes.

\section{Hypertension and Median Nerve Function}

Nerve Conduction Velocities. Separate 2 Group (hypertensive, normotensive) ANCOVAs were performed on sensory and compound motor nerve conduction velocities, covarying for body mass index, age and sex. There were no group differences in either the sensory or compound motor median nerve conduction velocities (see Table 2).

Sensory Action Potential Amplitudes. Separate 2 Group ANCOVAs performed on the amplitude of sensory action potentials, covarying for body mass index, age, sex, finger circumference and distance between stimulation and recording sites, revealed significant group differences for both wrist and elbow stimulation, as shown in Table 2 and Figure 1. In both instances, hypertensives had smaller amplitude sensory action potentials than normotensives. 


\section{Hypertension and Cutaneous Sensory Thresholds}

A 2 Group ANCOVA performed on the grand mean cutaneous sensory thresholds, covarying for body mass index, age, and sex, indicated that cutaneous sensory thresholds were higher in the hypertensive group than the normotensive group (see Table 2).

\section{Sensory Action Potential Amplitudes and Cutaneous Sensory Thresholds}

Multiple regression analyses were performed to determine the association between sensory action potential amplitudes from wrist and elbow stimulation and sensory thresholds, while accounting for the possible moderating influence of putative confounders. Two hierarchical regression analyses were conducted. Sex, body mass index, age, finger circumference and distance between stimulation and recording sites were entered in the first step. Cutaneous sensory threshold was entered in the second step. Sensory action potential amplitudes were negatively associated with cutaneous sensory thresholds; for both wrist $(B=-11.52$, $95 \%$ CI for $B=-19.13$ to $\left.-3.90, \beta=-.33, t=-3.04, \Delta R^{2}=.10, p<0.005\right)$, and elbow $(B=-7.20$, 95\% CI for $B=-12.29$ to $-2.11, \beta=-.31, t=-2.85, \Delta R^{2}=.08, p<0.01$ ) sensory action potentials.

\section{Discussion}

The current study examined median nerve function and cutaneous sensibility in patients with essential hypertension who were free from pre-existing peripheral neuropathy and associated symptoms. The major finding of this study was that hypertensives had lower amplitude sensory action potentials than normotensives by approximately $20 \%$. Earlier studies which have investigated peripheral nerve function in medicated essential hypertensives did not 
measure sensory action potential amplitudes (Bridgman et al., 1973; Halar et al., 1978; Viskoper et al., 1971). Thus, the current study was the first to report blood pressure-related changes in sensory action potentials of the peripheral nerves. As the amplitude of sensory action potentials reflects the number of large diameter myelinated fibres synchronously depolarised in the vicinity of the active recording electrode (Buchthal \& Rosenfalck, 1966), a reduction may indicate axonal loss (Gilliatt, 1978). The observation that neither sensory nor compound motor median nerve conduction velocities were different between hypertensives and normotensives in the current study concurs with the findings of most previous research (Bridgman et al., 1973; Halar et al., 1978). As previous studies tested patients on antihypertensive medication (Bridgman et al., 1973; Halar et al., 1978; Viskoper et al., 1971), their null findings may be confounded by the beneficial effects of antihypertensive agents on nerve function (e.g., Malik et al., 1998). Regardless, the current finding of unaltered median nerve conduction velocities in our sample of unmedicated hypertensives suggests that hypertension does not affect the myelination of peripheral afferents.

The present study also found that hypertensives were less sensitive to cutaneous stimulation than normotensive controls by approximately 30\%. This observation is in line with a previous study which reported higher cutaneous thresholds in hypertensives than normotensives (Rosa et al., 1994), and concurs with a well established literature showing that hypertensives have reduced perception of pain (Ghione, 1996). Further, the novel finding that sensory action potential amplitudes were inversely related to cutaneous sensory thresholds suggests that sensory-perceptual deficits in hypertension may be mediated by changes in peripheral nerve function, and, more specifically, axonal degeneration. 
The mechanism underlying a possible hypertension-related axonal degeneration has yet to be established. However, the lack of autoregulatory capabilities in the peripheral nerve vascular system (Smith et al., 1977), means the peripheral nerves need adequate vascularisation to prevent nerve ischaemia and hypoxia (Low \& Tuck, 1984; Olsson, 1972). As such, structural and functional alterations in the microcirculation associated with hypertension (Mourad \& Laville, 2006) could cause nerve hypoxia leading to axonal neuropathy.

\section{Limitations}

A limitation of the current study was that only the median nerve in the dominant arm was tested. Future studies would do well to test bilaterally and in multiple peripheral nerves in order to confirm the present study’s intriguing preliminary findings. In addition, axonal degeneration is often characterised by 'dying-back' in the most distal segments of the nerve (Greenfield, 1954), and therefore, examination of lower limb nerves may be better placed to demonstrate the presence of mild hypertension-related axonal neuropathy. The current study only tested nerve function in the large myelinated afferents which convey sensation from mechanoreceptors. It would be better for future studies to perform a battery of sensory tests to examine the effect of hypertension on thinly myelinated and unmyelinated afferents. Finally, although potential participants were excluded if secondary forms of hypertension were indicated, e.g., diabetes, they were not screened for metabolic syndrome. However, a review of the non-fasting glucose levels in the hypertensives revealed that the vast majority were in the normal range, suggesting the absence of metabolic syndrome. Thus, only a small number of our patients may have had metabolic syndrome. As such, the presence of metabolic syndrome in a small number of the hypertensives is unlikely to have a major impact on the group differences in sensory action potential amplitudes observed. Overall, definitive 
conclusions about hypertension-related peripheral nerve function cannot be drawn from these preliminary findings, which should be interpreted with caution until larger scale studies have been conducted.

\section{Conclusions}

The finding of smaller sensory action potential amplitudes in hypertensives compared to normotensives suggests that hypertensives may suffer from a mild subclinical form of axonal neuropathy. Lower cutaneous sensibility in hypertensives compared to normotensives suggests that sensory deficits in hypertension are not exclusively nociceptive. That smaller sensory action potential amplitudes were associated with higher sensory thresholds suggests that known sensory-perceptual deficits in hypertensives may be due to peripheral neuropathy. These findings support the idea that hypertension may be a risk factor for neuropathy. 
Bolton, C. F. \& Carter, K. M. (1980). Human sensory nerve compound action-potential amplitude - Variation with sex and finger circumference. Journal of Neurology Neurosurgery and Psychiatry, 43, 925-928.

Bridgman, J. F., Bidgood, L., \& Hoole, R. (1973). Nerve-conduction velocity and hypertension. British Medical Journal, 3, 500-501.

Buchthal, F. \& Rosenfalck, A. (1966). Evoked action potentials and conduction velocity in human sensory nerves. Brain Research, 3, 1-122.

Buschbacher, R. M. (1998). Body mass index effect on common nerve conduction study measurements. Muscle \& Nerve, 21, 1398-1404.

Dyck, P. J., Karnes, J. L., Obrien, P., Okazaki, H., Lais, A., \& Engelstad, J. (1986). The spatial-distribution of fiber loss in diabetic polyneuropathy suggests ischemia. Annals of Neurology, 19, 440-449.

Edwards, L., Ring, C., McIntyre, D., Carroll, D., \& Martin, U. (2007). Psychomotor speed in hypertension: Effects of reaction time components, stimulus modality, and phase of the cardiac cycle. Psychophysiology, 44, 459-468.

Fazan, V. P. S., Fazan, R., Salgado, H. C., \& Barreira, A. A. (1999). Morphology of aortic depressor nerve myelinated fibers in normotensive Wistar-Kyoto and spontaneously hypertensive rats. Journal of the Autonomic Nervous System, 77, 133-139.

Fazan, V. P. S., Salgado, H. C., \& Barreira, A. A. (2001). Aortic depressor nerve unmyelinated fibers in spontaneously hypertensive rats. AJP - Heart and Circulatory Physiology, 280, H1560-H1564.

Forrest, K. Y. Z., Maser, R. E., Pambianco, G., Becker, D. J., \& Orchard, T. J. (1997). Hypertension as a risk factor for diabetic neuropathy - A prospective study. Diabetes, 46, 665-670.

Ghione, S. (1996). Hypertension-associated hypalgesia. Hypertension, 28, 494-504.

Ghione, S., Rosa, C., Mezzasalma, L., \& Panattoni, E. (1988). Arterial hypertension is associated with hypalgesia in humans. Hypertension, 12, 491-497.

Ghione, S., Rosa, C., Panattoni, E., Nuti, M., Mezzasalma, L., \& Giuliano, G. (1985). Comparison of sensory and pain threshold in tooth pulp stimulation in normotensive man and essential hypertension. Journal of Hypertension, 3, s113-s115.

Gilliatt, R. W. (1978). Sensory conduction studies in early recognition of nerve disorders. Muscle \& Nerve, 1, 352-359.

Greenfield, J. C. (1954). The spino-cerebellar degenerations. Oxford: Blackwell.

Halar, E. M., Stewart, D. T., Venkatesh, B., \& Chrissian, S. A. (1978). Nerve-conduction velocity in hypertensive patients. Archives of Internal Medicine, 138, 121-123. 
Horowitz, S. H. \& Krarup, C. (1992). Conduction studies of the normal sural nerve. Muscle \& Nerve, 15, 374-383.

Ibrahim, S., Harris, N. D., Radatz, M., Selmi, F., Rajbhandari, S., Brady, L. et al. (1999). A new minimally invasive technique to show nerve ischaemia in diabetic neuropathy. Diabetologia, 42, 737-742.

Jarmuzewska, E. A. \& Mangoni, A. A. (2005). Pulse pressure is independently associated with sensorimotor peripheral neuropathy in patients with type 2 diabetes. Journal of Internal Medicine, 258, 38-44.

Katims, J. J., Naviasky, E. H., Rendell, M. S., Ng, L. K. Y., \& Bleecker, M. L. (1987). Constant current sine wave trans-cutaneous nerve-stimulation for the evaluation of peripheral neuropathy. Archives of Physical Medicine and Rehabilitation, 68, 210-213.

Levitt, H. (1971). Transformed up-down methods in psychoacoustics. Journal of the Acoustical Society of America, 49, 467-477.

Low, P. A. \& Tuck, R. R. (1984). Effects of changes of blood-pressure, respiratory-acidosis and hypoxia on blood-flow in the sciatic-nerve of the rat. Journal of Physiology-London, 347, 513-524.

Makin, A., Lip, G. Y. H., Silverman, S., \& Beevers, D. G. (2001). Peripheral vascular disease and hypertension: a forgotten association? Journal of Human Hypertension, 15, 447-454.

Malik, R. A. (2000). Can diabetic neuropathy be prevented by angiotensin-converting enzyme inhibitors? Annals of Medicine, 32, 1-5.

Malik, R. A., Williamson, S., Abbott, C., Carrington, A. L., Iqbal, J., Schady, W. et al. (1998). Effect of angiotensin-converting-enzyme (ACE) inhibitor trandolapril on human diabetic neuropathy: Randomised doubleblind controlled trial. Lancet, 352, 1978-1981.

Mazzucchi, A., Mutti, A., Poletti, A., Ravanetti, C., Novarini, A., \& Parma, M. (1986). Neuropsychological deficits in arterial-hypertension. Acta Neurologica Scandinavica, 73, 619-627.

Mourad, J. J. \& Laville, M. (2006). Is hypertension a tissue perfusion disorder? Implications for renal and myocardial perfusion. Journal of Hypertension, 24, S10-S16.

Olsson, Y. (1972). The involvement of vasa nervorum in disease of peripheral nerve. In P.J.Vinkin \& G. W. Bruyn (Eds.), Handbook of Clinical Neurology (pp. 644-664). Amsterdam: North-Holland.

Reja, A., Tesfaye, S., Harris, N. D., \& Ward, J. D. (1995). Is ace-inhibition with lisinopril helpful in diabetic neuropathy. Diabetic Medicine, 12, 307-309.

Ring, C., France, C. R., Al'Absi, M., Edwards, L., McIntyre, D., Carroll, D. et al. Effects of naltrexone on electrocutaneous pain in patients with hypertension compared to normotensive individuals. Biological Psychology, (in submission). 
Rosa, C., Ghione, S., Panattoni, E., Mezzasalma, L., \& Giuliano, G. (1986). Comparison of pain perception in normotensives and borderline hypertensives by means of a tooth pulpstimulation test. Journal of Caiovascular Pharmacology, 8, s125-s127.

Rosa, C., Vignocchi, G., Panattoni, E., Rossi, B., \& Ghione, S. (1994). Relationship between increased blood pressure and hypoalgesia: additional evidence for the existence of an abnormality of pain perception in arterial hypertension in humans. Journal of Human Hypertension, 8, 119-126.

Sabbatini, M., Bellagamba, G., Vega, J. A., \& Amenta, F. (2001). Effect of antihypertensive treatment on peripheral nerve vasculature in spontaneously hypertensive rats. Clinical and Experimental Hypertension, 23, 157-166.

Sabbatini, M., Vega, J. A., \& Amenta, F. (1996). Peripheral nerve vascular changes in spontaneously hypertensive rats. Neuroscience Letters, 217, 85-88.

Schaumburg, H. H., Spencer, P. S., \& Ochoa, J. (1983). The aging human peripheral nervous system. In R.Katzman \& R. D. Terry (Eds.), The neurology of aging (pp. 111-122). Philadelphia: F.A.Davis.

Shapiro, A. P., Miller, R. E., King, E., Ginchereau, E. H., \& Fitzgibbon, K. (1982). Behavioral consequences of mild hypertension. Hypertension, 4, 355-360.

Smith, D. R., Kobrine, A. I., \& Rizzoli, H. V. (1977). Absence of autoregulation in peripheral-nerve blood-flow. Journal of the Neurological Sciences, 33, 347-352.

Tesfaye, S., Chaturvedi, N., Eaton, S. E. M., Ward, J. D., Manes, C., Ionescu-Tirgoviste, C. et al. (2005). Vascular risk factors and diabetic neuropathy. New England Journal of Medicine, 352, 341-350.

Tesfaye, S., Harris, N., Jakubowski, J. J., Mody, C., Wilson, R. M., Rennie, I. G. et al. (1993). Impaired blood-flow and arteriovenous shunting in human diabetic neuropathy - A novel technique of nerve photography and fluorescein angiography. Diabetologia, 36, 12661274.

Teunissen, L. L., Franssen, H., Wokke, J. H. J., van der Graaf, Y., Linssen, W. H. J. P., Banga, J. D. et al. (2002). Is cardiovascular disease a risk factor in the development of axonal polyneuropathy? Journal of Neurology Neurosurgery and Psychiatry, 72, 590-595.

Thomas, P. K. \& Lascelle, R. G. (1965). Schwann-cell abnormalities in diabetic neuropathy. Lancet, 1, 1355-1356.

Tomassoni, D., Traini, E., Vitaioli, L., \& Amenta, F. (2004). Morphological and conduction changes in the sciatic nerve of spontaneously hypertensive rats. Neuroscience Letters, 362, 131-135.

Viskoper, R. J., Chaco, J., \& Aviram, A. (1971). Nerve conduction velocity in assessment of hypertension. Archives of Internal Medicine, 128, 574-575.

Waldstein, S. R., Manuck, S. B., Ryan, C. M., \& Muldoon, M. F. (1991). Neuropsychological correlates of hypertension: Review and methodologic considerations. Psychological Bulletin, 110, 451-468. 
Williams, B., Poulter, N. R., Brown, M. J., Davis, M., McInnes, G. T., Potter, J. F. et al. (2004). Guidelines for management of hypertension: Report of the fourth working party of the British Hypertension Society, 2004 - BHSIV. Journal of Human Hypertension, 18, 139-185.

Wood, D., Durrington, P., McInnes, G., Poulter, N., Rees, A., \& Wray, R. (1998). Joint British recommendations on prevention of coronary heart disease in clinical practice. Heart, 80, S1-S29.

Zamir, N. \& Shuber, E. (1980). Altered pain perception in hypertensive humans. Brain Research, 201, 471-474.

Zarrelli, M. M., Amoruso, L., Beghi, E., Apollo, F., Di Viesti, P., \& Simone, P. (2001). Arterial hypertension as a risk factor for chronic symmetric polyneuropathy. Journal of epidemiology and biostatistics, 6, 409-413. 


\section{Author Notes}

This study and LE was supported by the British Heart Foundation (FS/03/128). 
TABLE 1. Mean (SD) Characteristics of the Hypertensive and Normotensive Groups as well as the Degrees of Freedom, F-values and Statistical Significance Level of the Group Effects and Associated Effect Size

\begin{tabular}{ccccccc}
\hline \multicolumn{1}{c}{ Variable } & Hypertensive & Normotensive & df & F & $\boldsymbol{p}$ & $\boldsymbol{\eta}^{2}$ \\
\hline Ambulatory (Daytime) & & & & & & \\
$\quad$ Systolic blood pressure (mmHg) & $149.4(6.4)$ & $118.4(9.5)$ & 1,56 & 214.65 & $<.001$ & .79 \\
$\quad$ Diastolic blood pressure (mmHg) & $97.0(7.4)$ & $76.9(6.3)$ & 1,56 & 124.38 & $<.001$ & .69 \\
Laboratory & & & & & & \\
$\quad$ Systolic blood pressure (mmHg) & $148.6(12.0)$ & $116.7(14.2)$ & 1,57 & 87.57 & $<.001$ & .60 \\
$\quad$ Diastolic blood pressure (mmHg) & $94.4(8.4)$ & $72.0(8.1)$ & 1,57 & 108.33 & $<.001$ & .65 \\
$\quad$ Heart rate (beats/min) & $78.6(11.3)$ & $68.8(11.0)$ & 1,57 & 11.44 & $<.001$ & .16 \\
Body mass index (kg/m $\left.{ }^{2}\right)$ & $27.8(4.3)$ & $25.2(3.5)$ & 1,57 & 6.33 & $<.05$ & .10 \\
Age (years) & $40.9(6.7)$ & $35.1(7.1)$ & 1,57 & 10.69 & $<.005$ & .15 \\
Alcohol intake (units/week) & $10.9(9.9)$ & $8.5(8.6)$ & 1,57 & 0.99 & .32 & .01 \\
\hline
\end{tabular}


TABLE 2. Mean (SE) Median Nerve Conduction Velocities, Sensory Action Potential Amplitudes and Cutaneous Sensory Thresholds of the Hypertensive and Normotensive Groups as well as the Degrees of Freedom, F-values and Statistical Significance Level of the Group Effects and Associated Effect Size

\begin{tabular}{|c|c|c|c|c|c|c|}
\hline Variable & Hypertensive & Normotensive & df & $\mathbf{F}$ & $p$ & $\eta^{2}$ \\
\hline \multicolumn{7}{|c|}{ Median nerve conduction velocities (m/s) } \\
\hline Sensory & $66.3(1.72)$ & $67.7(1.55)$ & 1,46 & .362 & .55 & .008 \\
\hline Motor & $57.4(1.11)$ & $58.4(0.98)$ & 1,45 & .454 & .50 & .010 \\
\hline \multicolumn{7}{|c|}{ Sensory action potential amplitudes $(\mu \mathrm{V})$} \\
\hline Wrist & $20.85(1.6)$ & $26.77(1.47)$ & 1,47 & 6.57 & $<.01$ & .123 \\
\hline Elbow & $11.33(1.08)$ & $14.54(0.94)$ & 1,43 & 4.56 & $<.05$ & .096 \\
\hline Cutaneous sensory thresholds (mA) & $0.67(0.05)$ & $0.51(0.05)$ & 1,54 & 4.50 & $<.05$ & .077 \\
\hline
\end{tabular}




\section{Figure Caption}

Figure 1. Grand average sensory action potential waveforms for the hypertensive and normotensive groups, elicited by electrocutaneous stimulation of the median nerve at the wrist. 


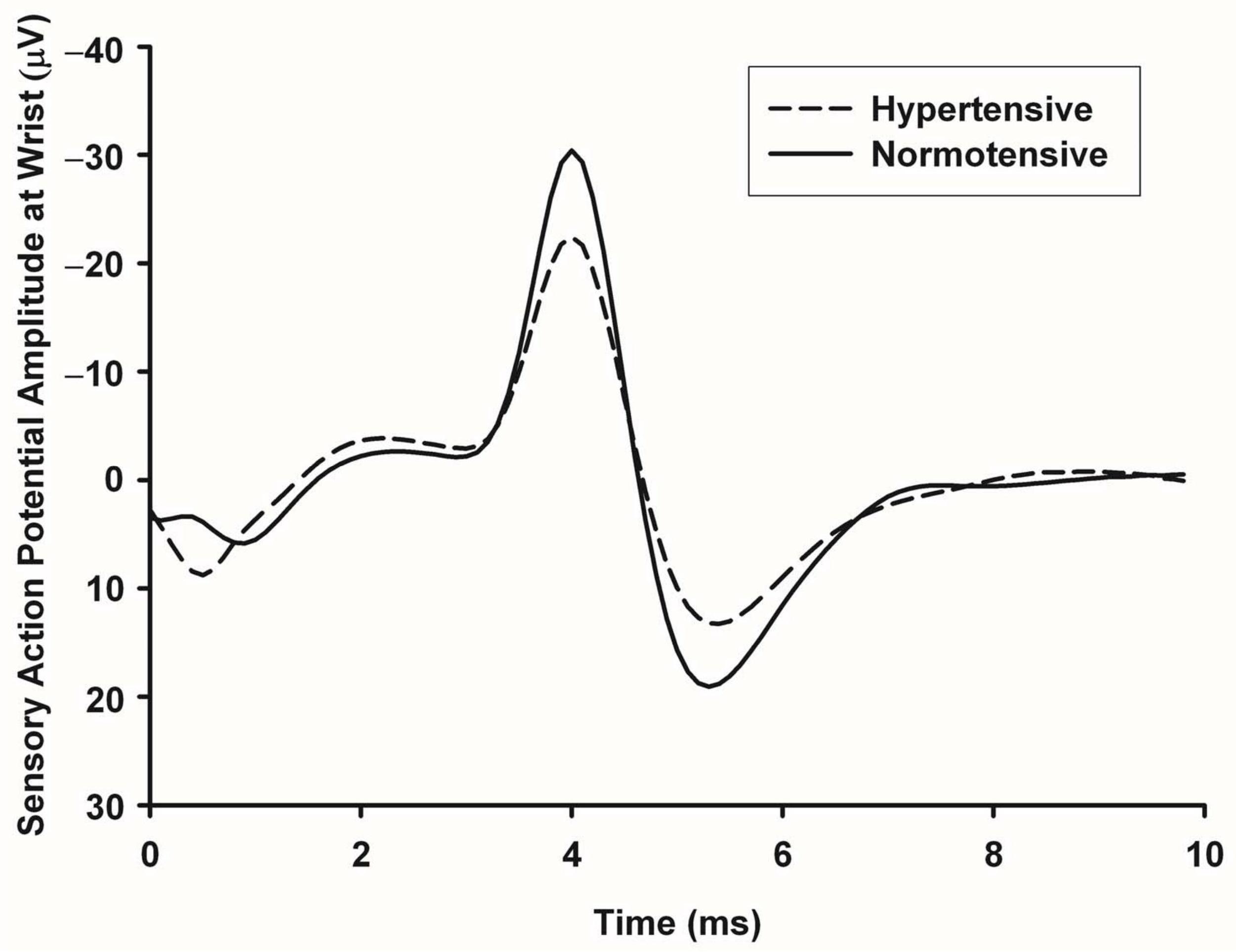

